\title{
A Review of Image Restoration based Image Defogging Algorithms
}

\author{
Bindu Bansal \\ Ph.D. Scholar, DAVIET, Jalandhar, INDIA \\ Email: er_bindu18@rediffmail.com \\ Dr. Jagroop Singh Sidhu \\ Associate Professor, DAVIET, Jalandhar, INDIA \\ Email: roopsidhu@yahoo.com \\ Dr. Kiran Jyoti \\ Associate Professor, GNDEC, Ludhiana, INDIA \\ Email: kiranjyotibains@yahoo.com
}

Received: 03 July 2017; Accepted: 15 August 2017; Published: 08 November 2017

\begin{abstract}
Haze and fog lead to image degradation by various degradation processes like image contrast, image blurring and pixel distortion. It has effected the efficiency of computer and machine vision algorithms. A number of single image and multiple image restoration based image defogging algorithms have aimed to solve the problem in an efficient and fast manner. The objective of the paper is to summarize present state of the art image defogging algorithms. Firstly, an image classification algorithm has been presented and then we summarized present state of the art image restoration based image defogging algorithms. Finally, we summarized image quality assessment methods followed by their comparisons of various image defogging algorithms. Problems of image dehazing and future scope have been discussed thereafter.
\end{abstract}

Index Terms - Image Dehazing, Dark Channel Prior, Image Restoration Methods, Image Quality Assessment, Image quality Assessment

\section{INTRODUCTION}

Bad Weather: Particles in Space: Weather conditions vary mainly in the types and sizes of the particles included and their concentrations in space [1]. TABLE 1 depicts the measure of particle sizes and concentrations under a variety of conditions. Larger particles produce a variety of weather conditions which are given below:

Table I. Measure of Particle Size

\begin{tabular}{cccc}
\hline Condition & Particle type & Radius $(\mu \mathrm{m})$ & $\begin{array}{c}\text { Concentration } \\
\left(\mathrm{cm}^{-3}\right)\end{array}$ \\
\hline Air & Molecule & $10^{-4}$ & $10^{19}$ \\
Haze & Aerosol & $10^{-2}-1$ & $10^{3}-10$ \\
Fog & Water droplet & $1-10$ & $100-10$ \\
Cloud & Water droplet & $1-10$ & $300-10$ \\
Rain & Water drop & $10^{2}-10^{4}$ & $10^{-2}-10^{-5}$ \\
\hline
\end{tabular}

Haze. Particle type of haze is aerosol which are small particles suspended in the gas. These particles are larger than air molecules but are smaller than fog droplets. Haze tends to create a distinctive gray or bluish hue and it affects visibility.

Fog. Fog evolves when the relative humidity of an air parcel reaches saturation point. A practical difference between fog and haze lies in the significantly reduced visibility caused by the former. There are many types of fog (e.g., radiation fog, advection fog, etc.) which differ from each other in their formation processes. In this paper, we will use both the terms interchangeably.

Cloud. A cloud exists on the higher altitudes. This is the real difference between fog and haze as cloud exists only at higher altitudes rather than sitting at ground level. Most clouds are made up of water droplets like fog, and some are composed of long ice crystals and ice-coated dust grains.

Rain and snow. When cloud droplets are processed, they turn into rain. When viewed up close, rain causes random spatial and temporal variations in images and hence must be dealt with differently. Similar arguments apply to snow, where the flakes are rough and have more complex shapes and optical properties. Details of formation of rain and snow are not discussed here.

Atmospheric Scattering [2] : As said above, air light or haze is the brownish or bluish color we see when we look at distant objects. This visible effect is due to the scattering of light by the atmosphere towards the camera. The atmosphere is a mixture of molecules and particles of various sizes. The following figure illustrates what happens when we take a picture of distant objects through the atmosphere during the daytime

On a bright sunny day, the primary illumination is the sun. This illumination is scattered by molecules and particles in the atmosphere in all different directions. Some of it (scattered light) is scattered towards the 


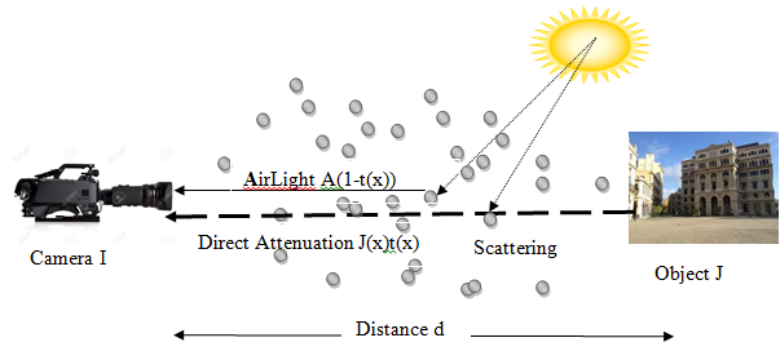

Fig.1. To view a distant object through atmosphere

viewer i.e. the camera which adds in an additive radiance that increases the brightness of distant objects. This is known as air light. The further the object is, the brighter it appears. It is because the more atmospheric medium is there between a viewer and an object; the more scattering of light towards the viewer takes place. The object radiance is also attenuated because of scattering and absorption as it travels towards the viewer. The direct transmission is the light radiance that remains after the object radiance is attenuated along the path towards the camera or the viewer.

\section{RELATED WORKS}

In this section, a review on classification of the input image, image dehazing methods image enhancement methods and image restoration methods has been made.

\section{A. Classification of Input Image}

The very first step is to consider whether the color input image is hazy or non-hazy. For this classification, one cannot depend on the human visual perception. An algorithm to judge whether the color input image is hazy or non-hazy has been proposed by R.K. Thakur et al. in [3]. In this paper, he has considered four parameters i.e. standard deviation, max, min and mean to categorize the images and for further classification, obtaining a high rate of recognition. He categorized the colored hazy images in four distinct groups as:

a) Non hazy color image

b) Slight hazy color image

c) Medium hazy color image

d) Extreme hazy color image

The author evaluated a parameter Visibility Index (VI) which is as follows:

$$
V I=\frac{\left(\sqrt{s d} *\left(\text { pixel_range }^{2}\right)\right.}{\left(\text { mean }^{2} 100\right)}
$$

where $s d=$ standard deviation of image mean $=$ mean of image

pixel_range $=\max -\min$

$\max =$ maximum intensity level in the image

$\min =$ minimum intensity level in the image
Then depending on the value of VI, the color input image is further categorized as follows:

Table 2. Visibility Index (VI) Values

\begin{tabular}{|ccc|}
\hline Category & $\begin{array}{c}\text { Value of Visibility Index } \\
(\mathrm{VI})\end{array}$ & \begin{tabular}{c} 
Type of Hazy Image \\
\hline 1
\end{tabular} \\
\hline $\mathrm{VI}>22$ & Non Hazy Image \\
\hline 2 & $14<\mathrm{VI} \leq 22$ & Slight Hazy Image \\
\hline 3 & $5<\mathrm{VI} \leq 14$ & Medium Hazy Image \\
\hline 4 & $0<\mathrm{VI} \leq 5$ & Extreme Hazy Image \\
\hline
\end{tabular}

The method is very simple, feasible, and efficient and classification accuracy achieved is $92 \%$ [3]. The method suffers from the limitation that it is not capable of classifying all types of noisy images. It mainly focuses on classification of color hazy image

\section{B. Image Dehazing[4]}

The presence of fog or haze in the atmosphere leads to reduce the visibility of the image scene substantially, and it has become a major problem. High-quality images are need of the hour as the many visual application systems like video surveillance, remote sensing; navigation, target identification are built from clear and high-quality images Updating the existing hardware equipment which can operate in adverse weather conditions needs significant work and high cost which is not realistic. On the other hand, it is more feasible to design haze removal method. Although many proposed image de-hazing methods have achieved good results, these methods still have some shortcomings regarding image quality achieved and computing speed. Therefore, image de-hazing is an urgent problem to be solved at present.

In some review papers, image de-hazing methods are divided into two categories on the basis of image enhancement and image restoration. The categories of image defogging methods are shown in figure 2 below. Table 3 shows the comparison of various single image dehazing techniques.

\section{Image Enhancement Methods}

These methods do not consider the physical imaging model of the hazy conditions. These methods improve the quality of image mainly by image contrast enhancement or color enhancement, and many algorithms have already been done using this criterion, such as histogram equalization algorithm [5] and multi-scale color image enhancement algorithm [6]. But this method may lead to loss of original part information, and the result of dehazing is distorted.

\section{Image Restoration Methods}

These methods use a physical imaging model based on the degradation reason of images under hazy conditions. These algorithms establish the atmospheric scattering model[7] which uses parameters like atmospheric light and transmission map(depth). The restored image is obtained by inversely solving the imaging model. The main problem with this method is that the atmospheric scattering model is an underdetermined equation, and 
another auxiliary information is needed to recover the foggy images[4].

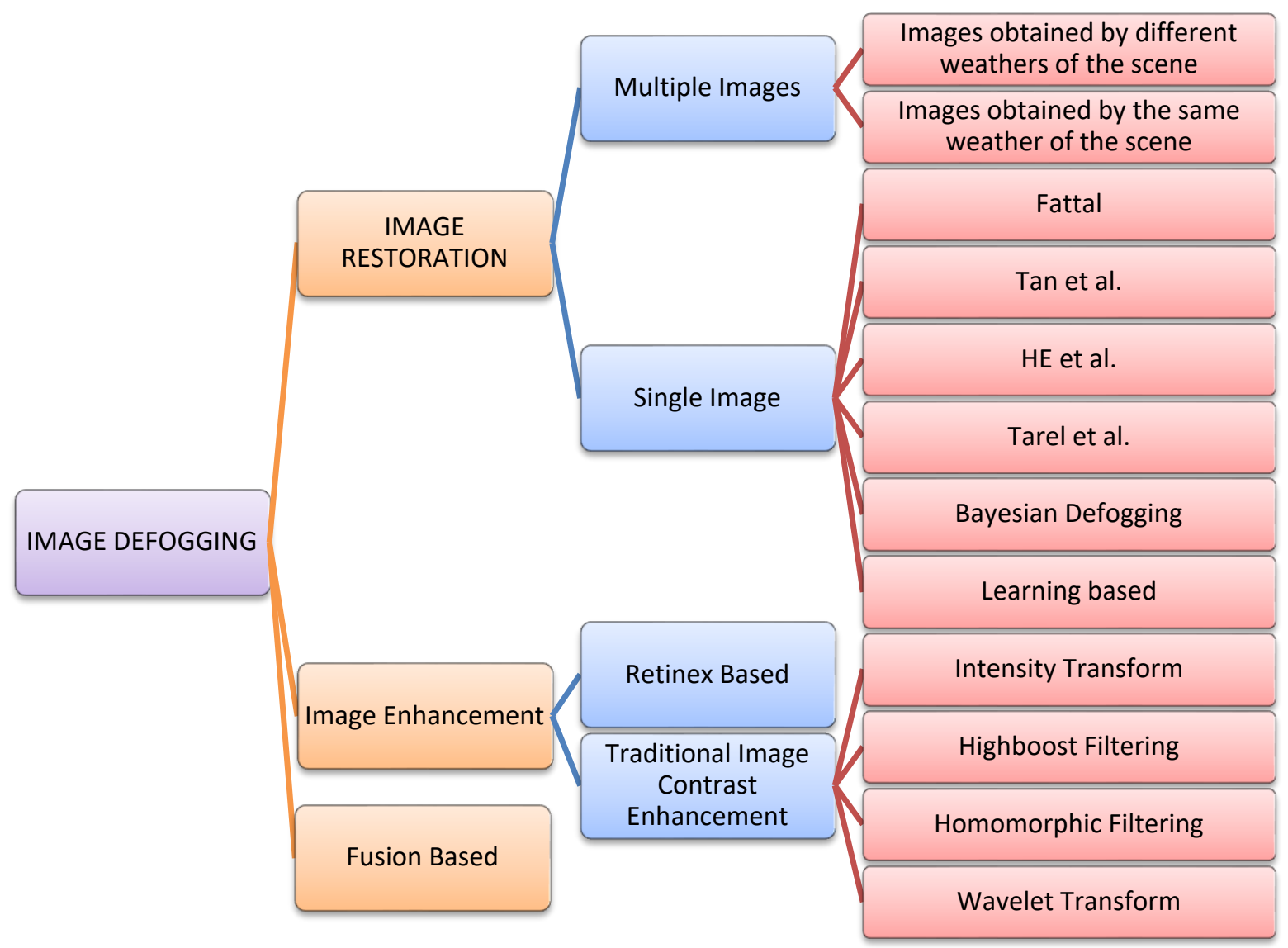

Fig.2. Classification of image dehazing techniques

To solve this problem many methods have already been proposed:

\section{Methods that use multiple foggy images:}

These methods further have two implementations. The first takes into account the multiple hazy images obtained under same weather conditions of the scene. The second uses the multiple images of the same scene taken under different weather conditions.

Same weather Conditions Multiple image methodology: because the air light scattered by atmospheric particles is partially polarised, many authors have designed image defogging algorithms using multiple polarising images. Many research papers based on this method include[8][9][10][11][12]. The polarization images with different brightnesses of the same circumstance of the scene are obtained by using a polarization filter with different orientations. This type of image restoration algorithms uses at least two polarization images to estimate the parameters of the physical model and then inversely solves the physical model for image restoration[13]. These polarization-based defogging algorithms are based on the partial polarization of air light. Its effect will decrease as the polarization degree decreases. Moreover, it will fail in thick foggy weather. Also, for some special cases like taking images with a moving camera, it was difficult to acquire two polarization images since the scenes change more rapidly than the filter rotation.

Different weather Conditions Multiple image methodology:

Narasimhan et al. have made a lot of effort on image restoration algorithm by using two or more images which were obtained under different weather conditions [14] [1] [15] [16] [7] [17]. The author has analysed the visual manifestations of different weather conditions, and then presented a physical dichromatic atmospheric scattering model [18]. Regarding this model, they presented a geometric framework for scene understanding under the foggy weather and computed the three-dimensional structure and color of the scene from two or more hazy images [16]. But if the color of the object in the scene matches fog or haze, it is unstable for defogging using this model. To solve the problem, a monochrome atmospheric scattering model was presented [7]. Narasimhan et al. also presented a fast image defogging algorithm based on this model [7]. Above image restoration algorithms are suitable for surveillance scenes only. For dynamic scenes especially for vehicle cameras, these algorithms fail because the two images are hard to be simultaneously acquired. 


\section{Methods that use Single foggy image:}

Single image defogging algorithms can enhance hazy images captured under any condition. To improve the efficiency of single image dehazing, several methods have been proposed till date:

Image Restoration Algorithm by Fattal's work:

Based on the assumption that the propagation of light and shading parts of the target surface are locally uncorrelated, Fattal [19] presented a method to estimate the transmission and atmospheric light. The author used independent component analysis (ICA) to estimate the medium transmission and then recovered the foggy images. This defogging method uses the statistical property to estimate parameters for image restoration. Thus, the performance greatly depends on the input image. This approach fails when the fog is dense, and there is insufficient signal-to-noise ratio (SNR).

Image Restoration Algorithm by Tan et al.:

Through statistics, Tan [20] made two basic observations: firstly that clear images had higher contrast compared with foggy images, so he focused on maximizing the local contrast of the recovered image for enhancing image visibility. Secondly, airlight changes smoothly in a small local area. To achieve the objectives, he first used the white balanced operation to transform the input image into white color. He then used the Markov Random Field (MRF) to model the airlight model with which the airlight could be estimated via maximizing the local contrast of the restored image. The purpose of this methodology is to obtain a restored image which has maximum contrast. This method does not take into account color restoration, which gives color distortion to the enhanced image. So the disadvantage is that the color of the restored image is often oversaturated, and partial information may lose. Also, as the patchbased operation is used to estimate the airlight model, some 'halo' effect also appear in the resulting image especially in depth discontinuities areas.

Image Restoration Algorithms by $\mathrm{He}$ et al. and its variant techniques:

To solve the problems, arising from the above methods, $\mathrm{He}$ et al. proposed a defogging algorithm based on a single image, and it has proved to be an effective method to restore outdoor images[21]. They proposed a Single image haze removal method based on dark channel prior (DCP) and obtained impressive and good quality results. According to him, most local patches in haze-free outdoor images contain some pixels which have low intensities in at least one color channel[22]. Under this assumption, they estimate the unknown parameters in the haze physical model. The formal definition of the concept of DCP for a haze-free image $J$ is as follows:

$$
J^{d a r k}(x)=\min _{c}\left(\min _{y \in \Omega(x)}\left(J^{c}(y)\right)\right)
$$

Where $J^{c}$ is a color channel in $\mathrm{J}$ and $\Omega(\mathrm{x})$ is a local patch centered at x. He et al., 2009 observed that for the non-sky region the dark channel of the haze-free image is low and very near to the null pixel value. Also, he noticed the fact that the dark channel is a rough approximation of the thickness of the scene.

The authors in [21] further investigated that the low intensities in the dark channel are due to 3 factors:

i. Shadows: For instance the shadow of cars, building or the shadows of leaves, trees and rocks in the landscape image.

ii. Colorful objects and/or surfaces: For instance any object like green grass/tree/plant, red or yellow flower/leaf, lacking color in any color channel (RGB) will result in low values in the dark channel.

iii. Dark objects or surfaces: for instance dark tree trunk and/or stone.

We observe that natural outdoor images are usually full of shadows, colorful objects, and the dark channels of these images are really dark!

The algorithm proposed by $\mathrm{He}$ et al. is as follows:

i. Estimate the atmospheric light A: To estimate the atmospheric light, the author pick $0.1 \%$ of the brightest pixel in the dark channel pixels, then the pixel which has the highest value of chosen dark channel well approximate the atmospheric light.

ii. Estimate the transmission: First apply the min operator on each color channel by using the definition of haze physical model and by assuming $\tilde{t}^{\mathrm{t}}$ constant in a local patch:

Since the airlight contains the highest pixel values by applying the min operator over the three color channels,

$$
\min _{c}\left(\min _{y \in \Omega(x)}\left(\frac{I^{c}(y)}{A^{c}}\right)\right)=\tilde{t}(x) \min _{c}\left(\min _{y \in \Omega(x)}\left(\frac{I^{c}(y)}{A^{c}}\right)\right)+(1-\tilde{t}(x))
$$

Property of dark channel on haze free image:

$$
J^{\text {dark }}(x)=\min _{c}\left(\min _{y \in \Omega(x)}\left(J^{c}(y)\right)\right)=0
$$

Which implies that the patch transmission is written as follows:

$$
\tilde{t}(x)=1-\min \left(\min \left(\frac{I^{c}(y)}{A^{c}}\right)\right)
$$

Here $w=0.95$ is assigned as the small amount of haze, allowing the aerial perspective.

iii. Define the transmission by soft matting i.e. solve the linear equation for $\mathrm{t}$

$$
(L+\lambda U)=\lambda \tilde{t}
$$

The technique is well defined in [23]. 
iv. Recover the scene radiance $\mathrm{J}$ by:

$$
\min _{y \in \Omega(x)}\left(\boldsymbol{I}^{c}(y)\right)=\tilde{t}(x)\left(\min _{y \in \Omega(x)}\left(\boldsymbol{J}^{c}(y)\right)\right)+(1-\tilde{t}(x)) A^{c}
$$

This step is just an application of the formula once the two parameters are allowing the recovering of the scene radiance $\mathrm{J}$, are estimated.

The more various color information the foggy image has, the better the restoration effect will be. But the DCP theory fails if the image has a large sky area, largely white area, or dense fog and inhomogeneous fog.

However, this method needs to refine the transmission map through soft matting technique which is computationally intensive and cannot be used in practical situations.

Huang et al. analyzed the drawback of the HE et al.'s DCP defogging algorithm and proposed an improved DCP (IDCP) algorithm with three modules for single image defogging[24]. The proposed algorithm can also enhance sandstorm images.

To improve the efficiency, Xie et al. used the Multiscale Retinex algorithm to estimate the transmission[6].

Gibson et al. used median filtering to optimize the transmission[25][26]. In addition, they also concluded that performing the defogging algorithm before image compressing is better than defogging after compressing[26]. But using median filtering causes edge degradation.

A number of different filters, which have good performance in preserving edge information, have also been used to replace the soft matting algorithm. Various research paper based on these filter use includes the weighted least square (WLS) based edge-preserving smoothing method[27], locally adaptive Wiener filter[28], bilateral filtering[29], and joint bilateral filtering[30].

To solve this problem of computation in the $\mathrm{HE}$ et al. [21] proposed a guided filter[31], and found that the output of a guided filter is an approximate solution of the Laplacian matting optimization equation. Also, Refining the transmission map using guided filter greatly reduces the time complexity. This method is proved to have a better edge preservation effect and was faster than bilateral filtering and joint bilateral filtering. But it suffers from the drawback that the original foggy image is chosen as the guidance image due to which the texture details of the recovered image are weakening and the haze removal results may not be the best. Another version of guided filter [31]was proposed by $\mathrm{He}$ et al. in [32] which improves the computational time.

Some improved defogging algorithms were further proposed on the basis of the guided image filtering. These includes Pei et al. used the DCP theory and guided image filtering to restore the night-time haze image[33], Li et al. [34] proposed a weighted guided image filter (WGIF) which addresses the problem that amplification of noise in sky regions is addressed for Single image haze removal.

$\mathrm{Li}$ and Zheng [35] also proposed a single image haze removal algorithm by introducing an edge-preserving decomposition technique to estimate the transmission map for a hazy image.

Lin and Wang [36] first used downsampling algorithm to resize the transmission and then used guided image filtering to optimize the transmission. Using the edgepreserving filters to replace the soft matting algorithm for image defogging not only significantly improves the efficiency, but also obtains a better edge restoration effect.

Zhu et al. [37] proposed a simple but powerful color attenuation prior to single image haze removal by creating a linear model to model the scene depth of the hazy image. Under this novel prior method with a supervised learning method, the depth information can be well recovered.

\section{E. Restoration Algorithm by Tarel et al.}

Tarel and Hautiere [38] proposed a fast image dehazing algorithm based on atmospheric veil by using median filtering and its variant to replace the soft matting algorithm. The algorithm can process real-time process color or gray images. This algorithm estimates atmospheric veil from the minimum color component using a median filter. For each pixel, it was assumed that the atmospheric veil $\mathrm{V}(\mathrm{x})$ was less than the minimal component of the original foggy image. Tarel et al. wanted to obtain the maximum $\mathrm{V}(\mathrm{x})$ which is smooth in most areas except for the edge area of the image. To improve the efficiency while preserving the edge and corner, they used the median filtering and its variant. This algorithm is very efficient, but since the median filter is not conformal and edge-preserving, the estimating atmospheric veil does not reflect the depth information of the scene accurately. As a result, in some small edge regions with large depth jumps, the desirable dehazing results cannot be achieved. The sky region processed by these algorithms is degraded by serious noise and color distortion.

\section{F. Image Restoration based on Bayesian Defogging [39]} [40]

Based on the fact that the scene albedo $\rho$ and depth $\mathrm{d}$ are two statistically independent components, authors Kratz and Nishino proposed a Bayesian defogging algorithm. They first factorized the image into the scene albedo and depth and then a Factorial Markov Random Field (FMRF) was applied to model the dependence between these two items and the input image. These algorithms are effective in reducing the halo artifacts, but the iteration is time-consuming, and the parameters need to be set manually.

\section{G. Learning-based Image Restoration Algorithm}

Tang et al. [41] proposed a novel transmission estimation method via a learning-based approach.

They used the Random Forest to learn a regression model. For an unknown foggy image, it uses three steps:

Step 1: The image is first divided into several small patches.

Step 2: Haze-relevant features are extracted. 
Step 3: The learned Random Forest model is used to obtain the transmission of each image patch.

Step 4: After that, the coarse transmission is obtained by aggregating the transmission of each image patch.

The algorithm also uses the guided image filtering to optimize the transmission further.

These algorithms have the ability to learn adaptive regression models for different weather conditions, which can restore the foggy image within homogeneous fog or dense fog.
These methods suffer from many shortcomings. It is hard to obtain a large number of training data as this algorithm needs many fog-free and foggy image pairs as training data for learning the regression model.

The coarse transmission which is estimated by the regression model is not the true transmission of the image.

It cannot reveal the true depth information of the image especially edge areas.

Table 3. Comparison of Various Single Image Dehazing Techniques

\begin{tabular}{|c|c|c|c|c|c|c|c|}
\hline $\begin{array}{l}\text { Defogging } \\
\text { Method }\end{array}$ & $\begin{array}{l}\text { Known } \\
\text { Parameters } \\
\text { (Input) }\end{array}$ & & $\begin{array}{l}\text { Estimating } \\
\text { (Output) }\end{array}$ & Key idea & Advantage & Disadvantage & Applications \\
\hline Tan 2008 & $\begin{array}{l}\text { Single } \\
\text { image } I(x)\end{array}$ & RGB & $\boldsymbol{L} \infty, \mathrm{t}(\mathrm{x}), \mathrm{R}(\mathrm{x})$ & $\begin{array}{l}\text { Brightest value assumption } \\
\text { for Atmospheric light } L \infty \\
\text { estimation } \\
\text { Maximal contrast assumption } \\
\text { for Scene reflection } \mathrm{R}(\mathrm{x}) \\
\text { estimation }\end{array}$ & $\begin{array}{l}\text { Good Contrast of } \\
\text { the Foggy Image }\end{array}$ & $\begin{array}{l}\text { Gives halo effect } \\
\text { and color } \\
\text { distortion as the } \\
\text { image is over } \\
\text { restored }\end{array}$ & $\begin{array}{l}\text { Single color or } \\
\text { gray foggy image }\end{array}$ \\
\hline Fattal 2008 & $\begin{array}{l}\text { Single } \\
\text { image } I(x)\end{array}$ & RGB & $\boldsymbol{L} \infty, \mathrm{t}(\mathrm{x}), \mathrm{R}(\mathrm{x})$ & $\begin{array}{l}\text { Shading and transmission are } \\
\text { locally and statistically } \\
\text { uncorrelated }\end{array}$ & $\begin{array}{lr}\text { High } & \text { image } \\
\text { visibility } & \text { in } \\
\text { homogenous } & \text { or } \\
\text { thin fog } & \end{array}$ & $\begin{array}{l}\begin{array}{l}\text { Fails to enhance } \\
\text { dense } \\
\text { image }\end{array} \\
\text { Insufficient } \\
\text { ratio }\end{array}$ & $\begin{array}{l}\text { Single color foggy } \\
\text { image }\end{array}$ \\
\hline $\begin{array}{l}\text { He et al. } \\
2009\end{array}$ & $\begin{array}{l}\text { Single } \\
\text { image } I(x)\end{array}$ & RGB & $\boldsymbol{L} \infty, \mathrm{t}(\mathrm{x}), \mathrm{R}(\mathrm{x})$ & $\begin{array}{l}\text { Dark channel: outdoor } \\
\text { objects in clear weather have } \\
\text { at least one colour channel } \\
\text { that is significantly dark }\end{array}$ & $\begin{array}{l}\text { Output image is } \\
\text { just similar as } \\
\text { input image and } \\
\text { has good color } \\
\text { restoration effect }\end{array}$ & $\begin{array}{l}\text { Fails under } \\
\text { inhomogeneous } \\
\text { fog } \\
\text { Fails to restore } \\
\text { large sky area or } \\
\text { white area }\end{array}$ & 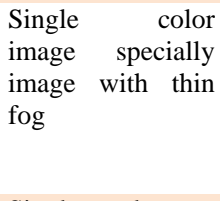 \\
\hline $\begin{array}{l}\text { Tarel } \quad- \\
\text { Hautiere } \\
2009\end{array}$ & $\begin{array}{l}\text { Single } \\
\text { image } I(x)\end{array}$ & RGB & $\boldsymbol{L} \infty, \mathrm{t}(\mathrm{x}), \mathrm{R}(\mathrm{x})$ & $\begin{array}{l}\text { Maximal contrast assumption } \\
\text { Normalized air light is upper- } \\
\text { bounded }\end{array}$ & $\begin{array}{l}\text { Fast } \\
\text { Good visibility for } \\
\text { thin image }\end{array}$ & $\begin{array}{l}\text { Color distortion } \\
\text { Fails to process } \\
\text { discontinuous } \\
\text { scene depth image }\end{array}$ & $\begin{array}{l}\text { Single color or } \\
\text { gray image }\end{array}$ \\
\hline $\begin{array}{lr}\text { Kratz } & - \\
\text { Nishino } & 2009\end{array}$ & $\begin{array}{l}\text { Single } \\
\text { image } I(x)\end{array}$ & RGB & $\mathrm{t}(\mathrm{x}), \mathrm{R}(\mathrm{x})$ & $\begin{array}{l}\text { Scene reflection } \mathrm{R}(\mathrm{x}) \text { and Air } \\
\text { light } \mathrm{A}(\mathrm{x}) \text { are statistically } \\
\text { independent } \\
\text { Layer separation }\end{array}$ & & & \\
\hline $\begin{array}{l}\text { Ancuti- } \\
\text { Ancuti } 2010\end{array}$ & $\begin{array}{l}\text { Single } \\
\text { image } I(x)\end{array}$ & RGB & $\mathrm{A}(\mathrm{x}), \mathrm{R}(\mathrm{x})$ & $\begin{array}{l}\text { Gray-world colour constancy; } \\
\text { Global contrast enhancement }\end{array}$ & & & \\
\hline $\begin{array}{l}\text { Meng et al. } \\
2013\end{array}$ & $\begin{array}{l}\text { Single } \\
\text { image } I(x)\end{array}$ & RGB & $\boldsymbol{L} \infty, \mathrm{t}(\mathrm{x}), \mathrm{R}(\mathrm{x})$ & $\begin{array}{l}\text { Dark channel for } \\
\text { transmission } t(x)\end{array}$ & $\begin{array}{l}\text { Gives good } \\
\text { contrast image }\end{array}$ & $\begin{array}{l}\text { Not suitable for } \\
\text { sky area or white } \\
\text { area } \\
\text { Over enhances the } \\
\text { image }\end{array}$ & $\begin{array}{l}\text { For single color } \\
\text { foggy image }\end{array}$ \\
\hline $\begin{array}{l}\text { Tang et al. } \\
2014\end{array}$ & $\begin{array}{l}\text { Single } \\
\text { image } I(x)\end{array}$ & RGB & $\mathrm{t}(\mathrm{x}), \mathrm{R}(\mathrm{x})$ & $\begin{array}{l}\text { Machine learning of } \\
\text { transmission } \mathrm{t}(\mathrm{x})\end{array}$ & & & \\
\hline Fattal 2014 & $\begin{array}{l}\text { Single } \\
\text { image } I(x)\end{array}$ & RGB & $\boldsymbol{L} \infty, \mathrm{t}(\mathrm{x}), \mathrm{R}(\mathrm{x})$ & $\begin{array}{l}\text { Colour line: small image } \\
\text { patch has uniform colour and } \\
\text { depth but different shading }\end{array}$ & & & \\
\hline $\begin{array}{l}\text { Cai et al. } \\
2016\end{array}$ & $\begin{array}{l}\text { Single } \\
\text { image } I(x)\end{array}$ & RGB & $\mathrm{t}(\mathrm{x}), \mathrm{R}(\mathrm{x})$ & $\begin{array}{l}\text { Learning of } \mathrm{t}(\mathrm{x}) \text { in } \mathrm{CNN} \\
\text { framework }\end{array}$ & $\begin{array}{l}\text { Better restores the } \\
\text { Sky and white } \\
\text { area }\end{array}$ & $\begin{array}{l}\text { Over enhances } \\
\text { dark colours }\end{array}$ & Single images \\
\hline $\begin{array}{l}\text { Berman et al. } \\
2016\end{array}$ & $\begin{array}{l}\text { Single } \\
\text { image } \mathrm{I}(\mathrm{x})\end{array}$ & RGB & $\mathrm{t}(\mathrm{x}), \mathrm{R}(\mathrm{x})$ & $\begin{array}{l}\text { Non-local haze line; finite } \\
\text { colour approximation }\end{array}$ & & & \\
\hline
\end{tabular}

\section{IMAGE QUALITY ACCESSMENT CRIETERIA}

The primary purpose of image defogging algorithms is to enhance the visibility of a hazy image. A good defogging algorithm not only enhances the visibility, edge, and texture information, but also preserves the image structure and its colors. Thus, a good image quality assessment method compares the effect of visibility, color restoration, and image structure similarity of different defogging algorithms.

\section{A. Assessment Criterion of Image Visibility}

Some of the indexes that can be used to compare the visibility of images are like blind assessment metric[42], image visibility measurement (IVM) [43], image contrast [44], and visual contrast measure (VCM) [45].

\section{Blind Assessment Indicator:}

It uses first two indicators (e and $\bar{r}$ ) to represent the enhanced degree of image visibility. 
e denotes for the increased rate of visible edges after image defogging and is calculated by

$$
e=\frac{n_{r}-n_{o}}{M \times N}
$$

where $n_{r}$ and $n_{o}$ represent the cardinal numbers of the set of visible edges in restored image and original image, respectively and $\mathrm{M}$ and $\mathrm{N}$ are the image size[42]. Larger the value of e, the larger degree of visibility improvement.

The second indicator $\bar{r}$ uses the enhanced degree of image gradients to represent the restoration degree of the image edge and texture information. A larger $\bar{r}$ also means that the corresponding defogging algorithm has better edge preservation performance than others.

$$
\bar{r}=\exp \left[\frac{1}{n_{r}} \sum_{i \in\{r} \log r_{i}\right]
$$

Where

$r_{i}=\frac{\Delta \boldsymbol{I}_{i}^{r}}{\Delta \boldsymbol{I}_{i}^{o}}, \Delta \boldsymbol{I}_{i}^{r}$, and $\Delta \boldsymbol{I}_{i}^{o}$ are the gradient of the restored image and original image, respectively, and $\wp \mathrm{r}$ denotes the set of visible edges of the restored image. This gradient based index can be used as an index to measure the restoration of edge information and to measure the restoration of edge information.

\section{Image Visibility Measurement (IVM):}

$\mathrm{Yu}$ et al. [43] presented another image visibility measurement method based on the visible edge segmentation.

$$
I V M=\frac{\boldsymbol{n}_{r}}{\boldsymbol{n}_{\text {total }}} \log \sum_{x \in \wp} C(x)
$$

Where

$n_{r}$ is the number of visible edges

$n_{\text {total }}$ is the number of edges,

$\mathrm{C}(\mathrm{x})$ is the mean contrast, and

$\wp$ denotes the image area of visible edges.

\section{Image Contrast:}

The contrast of a non-hazy image is usually much higher than that of a hazy image, so image contrast can be used to compare different defogging algorithms.

The higher the contrast of the enhanced image, the better is the dehazing algorithm.

Contrast gain is calculated as the mean contrast difference between the enhanced image and original foggy image [44], and is calculated as:

$$
C_{G A I N}=\overline{C_{E}}-\overline{C_{o}}
$$

Where $\overline{C_{E}}$ and $\overline{C_{o}}$ represent the mean contrast of the enhanced image and foggy image, respectively.

\section{Visual Contrast Measure (Vcm):}

Jobson et al. [45] proposed a visual contrast measure(VCM) to find the degree of the visibility of the image and is calculated by

$$
V C M=100 * \frac{\boldsymbol{R}_{V}}{\boldsymbol{R}_{T}}
$$

Where $R_{V}$ is the number of local areas whose standard deviation is larger than the given threshold and $\boldsymbol{R}_{T}$ is the total number of local areas.

\section{B. Assessment Criterion of Color Restoration}

Blind assessment indicator $(\sigma)$ is used to assess the color restoration performance of defogging algorithms[42]. Here, $\sigma$ denotes the rate of the saturated pixels after image defogging and is calculated as follows:

$$
\sigma=\frac{n_{s}}{M \times N}
$$

where $\mathrm{M}$ and $\mathrm{N}$ is the size of the image and $\mathrm{ns}$ is the total number of $\mathrm{B} \& \mathrm{~W}$ (black and white) pixels of the enhanced image (which are not completely B\&W in the original fog image). Smaller the value of $\sigma$, the better is the result of the defogging algorithm.

Also, by Yu et al., a good defogging algorithm should allow the original hazy image and enhanced image should have similar Histogram distributions. They used the Histogram Correlation Coefficient (HCC) of the two color images as the criterion to assess the performance of color restoration.

\section{Image Structure Similarity}

$\mathrm{Wu}$ and $\mathrm{Zhu}$ [46] used the image structural similarity(SSIM) and Universal Quality Index (UQI) [47] to assess the performance of the structural similarity between the original hazy image and the enhanced image. Both traditional SSIM and UQI criterions use an image with high quality as the reference image. Thus, the higher the SSIM and UQI, the better the compared image. Thus, in real-world applications of image defogging, the original hazy image is always chosen as the reference image, so large SSIM and UQI does not mean that the image is of high quality. So the enhanced image with the good/best visibility may have the smallest SSIM and UQI Also, the removal of fog from a foggy image will also change the image structure. This also leads to a small SSIM and UQI.

\section{Other Image Quality Crietrias}


Besides above said image quality criterions we used the other widely used image quality criterions also which includes the following:

i. $\quad$ Mean Squared Error

ii. Peak Signal to Noise Ratio

iii. Signal to Noise Ratio

iv. AverageDifference

v. MaximumDifference

vi. NormalizedAbsoluteError

vii. Normalized Cross Correlation(NK)

In the next section results of the various defogging algorithms along with their objective and subjective image quality criterions have been presented.

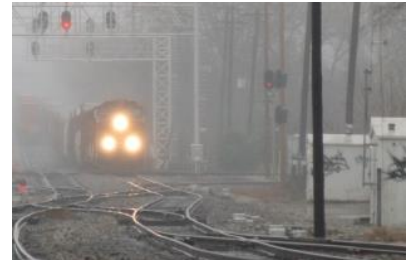

(a)

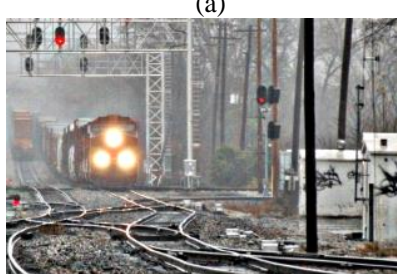

(e)

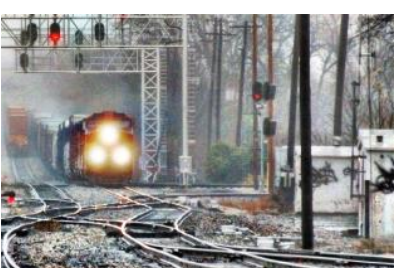

(b)

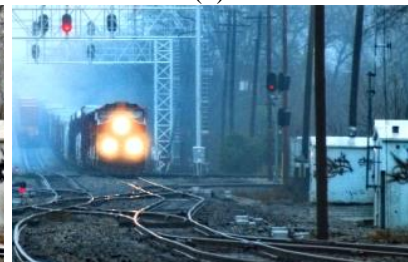

(f)

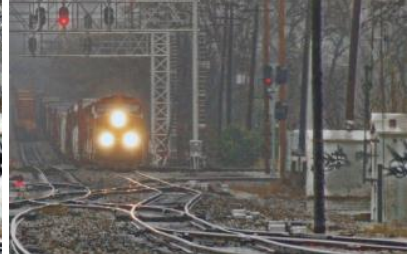

(c)

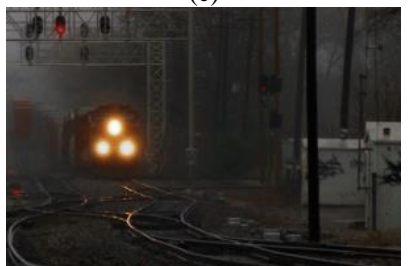

(g)

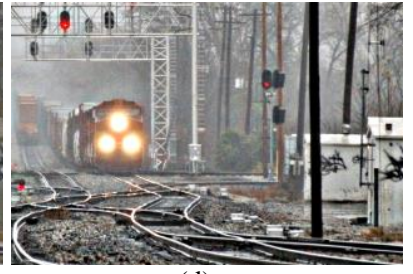

(d)

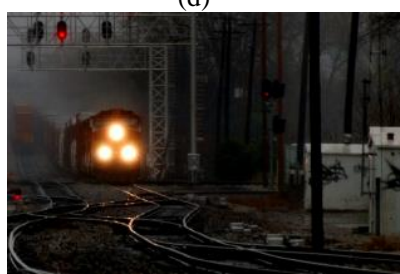

(h)

Fig.3. Results for the image "train.bmp" (a) Original Image (b) CLAHE Output (c) Tarel and Hautiere [38] (d) He et al. [31] (e) He et al. [32] (f) Meng et al. [48] (g) He et al. [21] (h) Cai et al.[49]

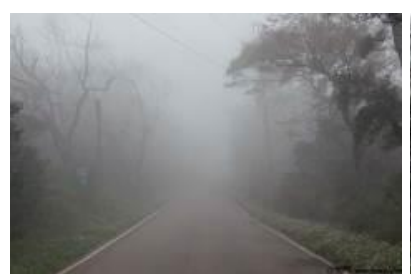

(a)

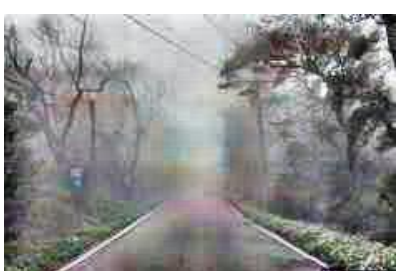

(b)

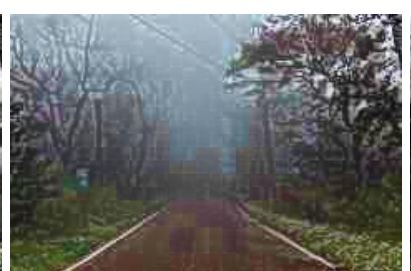

(c)

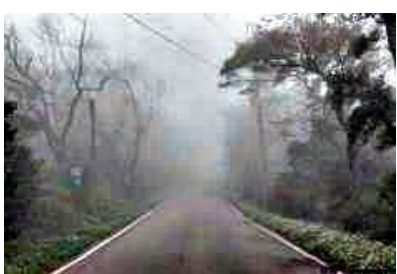

(d)

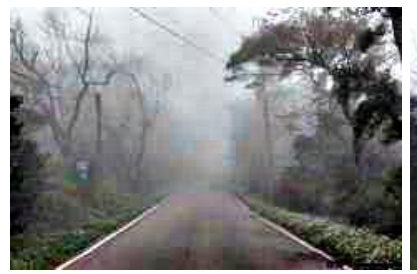

(e)

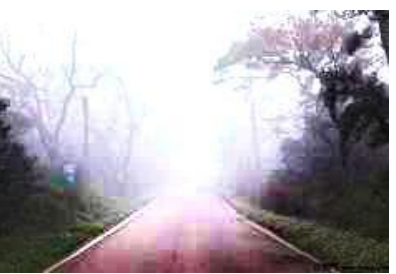

(f)

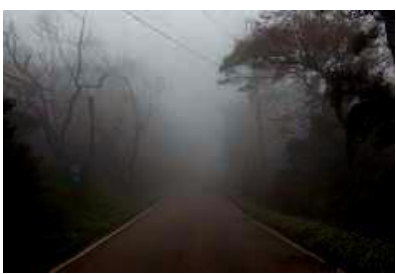

(g)

Fig.4. Results for the image "fog2.jpg” (a) Original Image (b) CLAHE Output (c) Tarel and Hautiere [38] (d) He et al. [31] (e) He et al. [32] (f) Meng et al. [48] (g) Cai et al.[49]

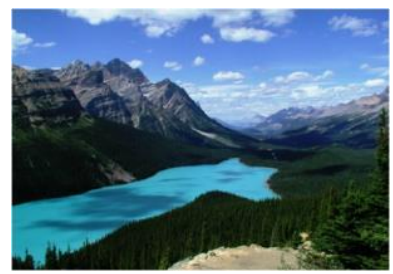

(a)

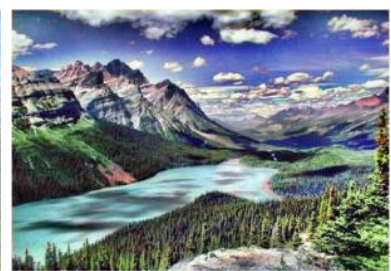

(b)

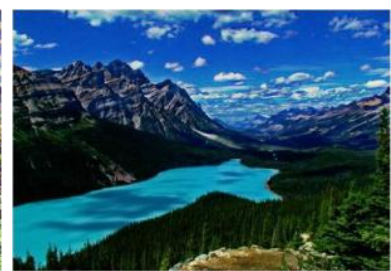

(c)

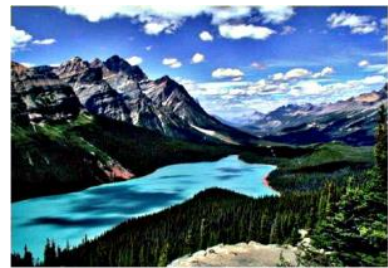

(d) 


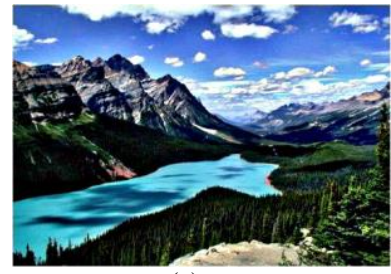

(e)

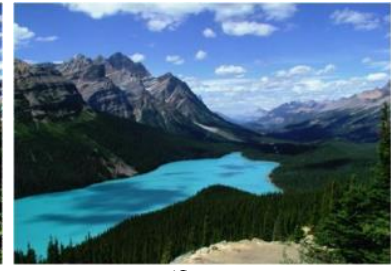

(f)

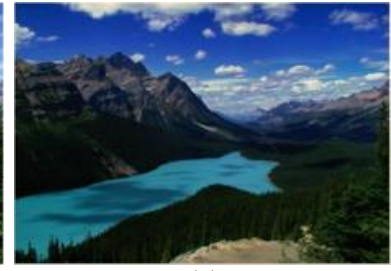

(g)

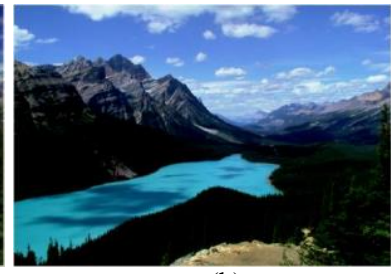

(h)

Fig.5.Results for the image "lake.jpg" (a) Original Image (b) CLAHE Output (c) Tarel and Hautiere [38] (d) He et al. [31] (e) He et al. [32] (f) Meng et al. [48] (g) He et al. [21] (h) Cai et al.[49]

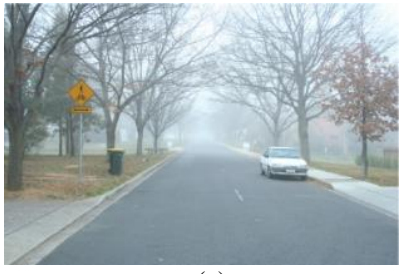

(a)

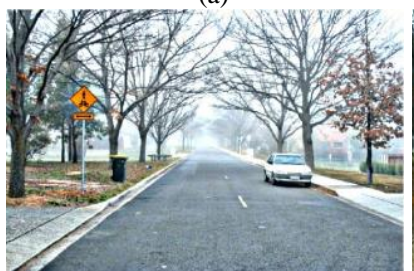

(e)

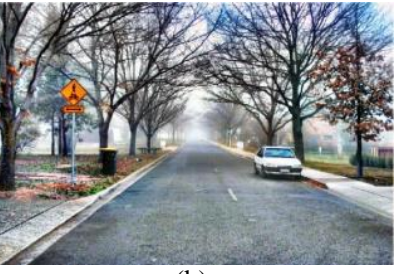

(b)

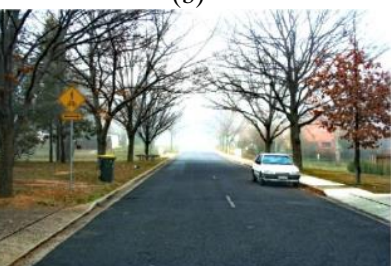

(f)

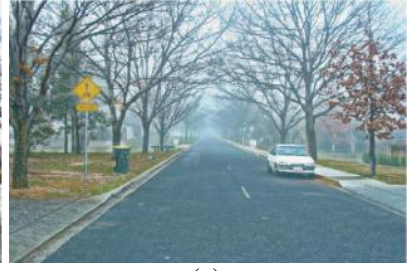

(c)

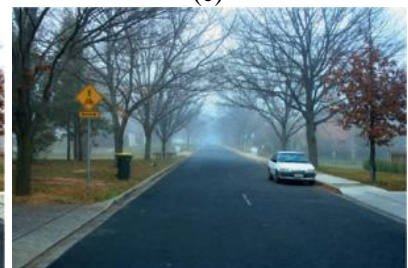

(g)

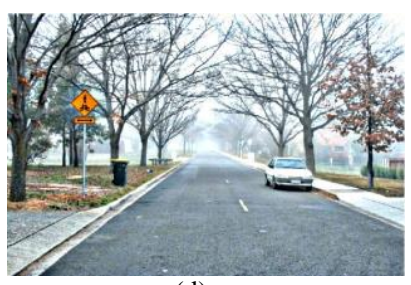

(d)

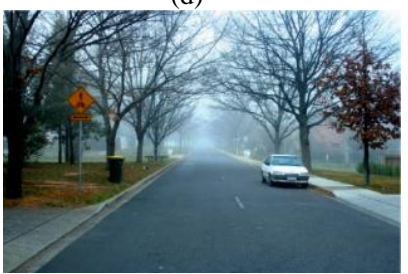

(h)

Fig.6. Results for the image "sweden.jpg" (a) Original Image (b) CLAHE Output (c) Tarel and Hautiere [38] (d) He et al. [31] (e) He et al. [32] (f) Meng et al. [48] (g) He et al. [21] (h) Cai et al.[49]

Table 4. Results For Visibility Index

\begin{tabular}{|c|c|c|c|}
\hline S.No. & Input Image & Visibility Index & Fog level \\
\hline 1 & Train.bmp & 20.3883 & Slight Hazy \\
\hline 2 & Fog2.jpg & 26.6002 & Non Hazy Image \\
\hline 3 & Lake.jpg & 58.7441 & Non Hazy Image \\
\hline 4 & Sweden.jpg & 23.1750 & Non Hazy Image \\
\hline
\end{tabular}

Table 5. Results For Fig.3 For Train.Bmp

\begin{tabular}{|c|c|c|c|c|c|c|c|}
\hline $\begin{array}{c}\text { Quality } \\
\text { Criteria }\end{array}$ & CLAHE & $\begin{array}{c}\text { Tarel and } \\
\text { Hautiere } \\
{[38]}\end{array}$ & $\begin{array}{c}\text { He et al. } \\
{[31]}\end{array}$ & $\begin{array}{c}\text { He et al. } \\
{[32]}\end{array}$ & $\begin{array}{c}\text { Meng et al. } \\
{[48]}\end{array}$ & $\begin{array}{c}\text { He et al. } \\
{[21]}\end{array}$ & Cai et al.[49] \\
\hline E & 26.0513 & 27.4954 & 20.8667 & 20.9329 & 21.0279 & 11.2967 & 23.0129 \\
\hline $\bar{r}$ & 4.4217 & 2.4996 & 3.8052 & 3.8045 & 2.4078 & 1.5482 & 1.3532 \\
\hline IVM & $9.7083 \mathrm{e}-004$ & 0 & 0.0395 & 0.0384 & 0.0048 & 0.0049 & 0.1259 \\
\hline $\begin{array}{c}\text { Contrast } \\
\text { Gain }\end{array}$ & 9.3180 & 8.7210 & 8.9182 & 8.9433 & 8.1589 & 5.7833 & 9.9342 \\
\hline VCM & 0.8173 & 0.2495 & 0.7431 & 0.7532 & 0.0905 & 0.7082 & -0.2298 \\
\hline$\Sigma$ & 0.5431 & 0.7796 & 0.5795 & 0.5795 & 0.7367 & 0.9153 & 0.3941 \\
\hline HCC & 0.4066 & 0.2867 & 0.4636 & 0.4647 & 0.3560 & 0.1437 & 1.2173 \\
\hline SSIM & 88 & 61 & 85 & 85 & 71 & 45 & 37.1667 \\
\hline UQI & 0.9225 & 0.8962 & 0.9281 & 0.9286 & 0.8737 & 0.9625 & 0.2253 \\
\hline MSE & $1.5862 \mathrm{e}+003$ & $1.6353 \mathrm{e}+003$ & $1.0379 \mathrm{e}+003$ & $1.0297 \mathrm{e}+003$ & $1.3253 \mathrm{e}+003$ & 396.2734 & $9.7754 \mathrm{e}+003$ \\
\hline PSNR & 16.1273 & 15.9950 & 17.9690 & 18.0036 & 16.9077 & 22.1509 & 8.2295 \\
\hline NK & 1.0242 & 0.7464 & 1.0266 & 1.0246 & 0.8833 & 0.9267 & 0.2988 \\
\hline AD & 1.9157 & 32.4341 & 0.2771 & 0.5028 & 21.3128 & 12.8247 & 96.9289 \\
\hline SC & 0.8813 & 1.7188 & 0.9011 & 0.9047 & 1.1922 & 1.1429 & 7.6967 \\
\hline MD & 111 & 80 & 117 & 115 & 89 & 52 & 137 \\
\hline NAE & 0.2477 & 0.2718 & 0.1876 & 0.1867 & 0.2502 & 0.1339 & 0.7348 \\
\hline & & & & & & & \\
\hline
\end{tabular}


Table 6. Results For Fig.4 For Fog2.Jpg

\begin{tabular}{|c|c|c|c|c|c|c|}
\hline $\begin{array}{c}\text { Quality } \\
\text { Criteria }\end{array}$ & CLAHE & $\begin{array}{c}\text { Tarel and } \\
\text { Hautiere [38] }\end{array}$ & $\begin{array}{c}\text { He et al. } \\
{[31]}\end{array}$ & $\begin{array}{c}\text { He et al. } \\
{[32]}\end{array}$ & $\begin{array}{c}\text { Meng et al. } \\
{[48]}\end{array}$ & Cai et al.[49] \\
\hline E & 19.5559 & 33.5363 & 17.8288 & 17.9843 & 12.9050 & 14.1493 \\
\hline $\bar{r}$ & 4.3271 & 4.1532 & 3.6463 & 3.6488 & 2.8985 & 1.0165 \\
\hline IVM & $3.4864 \mathrm{e}-004$ & 0 & 0.0042 & 0.0043 & 0.1762 & 0.0791 \\
\hline Contrast Gain & 5.3381 & 6.4799 & 5.1684 & 5.2491 & 4.6943 & 4.9751 \\
\hline VCM & 0.7964 & 0.5472 & 0.8307 & 0.8407 & -0.1557 & -0.1504 \\
\hline$\Sigma$ & 0.6135 & 0.6098 & 0.6820 & 0.6812 & 0.7089 & 0.6084 \\
\hline HCC & 0.3208 & 0.4237 & 0.4287 & 0.4307 & 0.2038 & 0.3660 \\
\hline SSIM & 47.1869 & 38.2940 & 38.6570 & 38.6570 & 58.6207 & 31.3975 \\
\hline UQI & 0.9404 & 0.8968 & 0.9455 & 0.9459 & 0.8379 & 0.4832 \\
\hline MSE & $1.0735 \mathrm{e}+003$ & $1.3383 \mathrm{e}+003$ & 634.5466 & 623.1465 & $4.4770 \mathrm{e}+003$ & $3.8182 \mathrm{e}+003$ \\
\hline PSNR & 17.8229 & 16.8652 & 20.1062 & 20.1849 & 11.6210 & 12.3122 \\
\hline NK & 1.0574 & 0.7926 & 1.0314 & 1.0255 & 1.3984 & 0.6084 \\
\hline AD & -11.5158 & 26.5609 & 0.0844 & 0.7941 & -44.0343 & 59.4747 \\
\hline SC & 0.8502 & 1.5109 & 0.9097 & 0.9203 & 0.4871 & 2.2963 \\
\hline MD & 130 & 76 & 103 & 104 & 69 & 87 \\
\hline NAE & 0.1981 & 0.2639 & 0.1522 & 0.1501 & 0.4679 & 0.4787 \\
\hline
\end{tabular}

Table 7. Results For Fig.5 For Lake.Jpg

\begin{tabular}{|c|c|c|c|c|c|c|c|}
\hline $\begin{array}{c}\text { Quality } \\
\text { Criteria }\end{array}$ & CLAHE & $\begin{array}{c}\text { Tarel and } \\
\text { Hautiere [38] }\end{array}$ & $\begin{array}{c}\text { He et al. } \\
{[31]}\end{array}$ & $\begin{array}{c}\text { He et al. } \\
{[32]}\end{array}$ & $\begin{array}{c}\text { Meng et al. } \\
{[48]}\end{array}$ & $\begin{array}{c}\text { He et al. } \\
{[21]}\end{array}$ & $\begin{array}{c}\text { Cai et } \\
\text { al.[49] }\end{array}$ \\
\hline $\mathrm{e}$ & 4.6335 & 8.4004 & 9.2779 & 9.3417 & 0.2260 & 1.4470 & -3.9759 \\
\hline $\bar{r}$ & 3.1588 & 1.4748 & 2.0329 & 2.0452 & 1.0973 & 1.1012 & 0.7509 \\
\hline IVM & $6.5156 \mathrm{e}-004$ & $5.9048 \mathrm{e}-004$ & 0.0246 & 0.0237 & $3.1899 \mathrm{e}-$ & 0.0020 & 0.1613 \\
& & & & & 004 & & \\
\hline Contrast Gain & 8.5930 & 8.4431 & 8.9103 & 8.9626 & 7.0053 & 7.0569 & 7.3025 \\
\hline VCM & -0.0356 & 0.4629 & 0.2602 & 0.2622 & 0.9572 & 0.5544 & 0.1724 \\
\hline$\Sigma$ & 0.5598 & 0.7857 & 0.6699 & 0.6684 & 0.9514 & 0.9486 & 0.6503 \\
\hline HCC & 0.1657 & 0.3204 & 0.7370 & 0.7353 & -0.0171 & 0.0138 & 0.2548 \\
\hline SSIM & 89.1071 & 60.8929 & 81.6071 & 81.7857 & 48.2143 & 54.1071 & 46.0714 \\
\hline UQI & 0.6850 & 0.7414 & 0.8396 & 0.8810 & 0.9577 & 0.9845 & 0.6053 \\
\hline MSE & $2.8577 \mathrm{e}+003$ & $1.5876 \mathrm{e}+003$ & 740.2930 & 739.3213 & 15.7200 & 69.7849 & 386.1237 \\
\hline PSNR & 13.5706 & 16.1233 & 19.4368 & 19.4425 & 36.1663 & 29.6932 & 22.2635 \\
\hline NK & 1.0696 & 0.7031 & 1.0573 & 1.0565 & 1.0018 & 1.0017 & 0.9151 \\
\hline AD & -24.6922 & 27.6305 & -1.6416 & -1.5955 & -0.8788 & 1.0941 & 15.9827 \\
\hline SC & 0.7349 & 1.8892 & 0.8532 & 0.8545 & 0.9951 & 0.9912 & 1.1625 \\
\hline MD & 114 & 131 & 98 & 101 & 31 & 44 & 62 \\
\hline NAE & 0.4657 & 0.3206 & 0.2284 & 0.2281 & 0.0298 & 0.0617 & 0.1727 \\
\hline
\end{tabular}

Table 8. Results For Fig.6 For Sweden.Jpg

\begin{tabular}{|c|c|c|c|c|c|c|c|}
\hline $\begin{array}{l}\text { Quality } \\
\text { Criteria }\end{array}$ & CLAHE & $\begin{array}{c}\text { Tarel and } \\
\text { Hautiere [38] }\end{array}$ & $\begin{array}{l}\text { He et al. } \\
\text { [31] }\end{array}$ & He et al. [32] & $\begin{array}{l}\text { Meng et al. } \\
\text { [48] }\end{array}$ & $\begin{array}{l}\text { He et al. } \\
\text { [21] }\end{array}$ & $\begin{array}{l}\text { Cai et } \\
\text { al.[49] }\end{array}$ \\
\hline $\mathrm{e}$ & 15.0371 & 13.7196 & 13.2700 & 13.3092 & 11.5542 & -0.0317 & 5.8892 \\
\hline $\bar{r}$ & 3.5084 & 2.0157 & 3.3710 & 3.3733 & 2.3897 & 1.5492 & 1.5836 \\
\hline IVM & 0.0017 & $1.2500 \mathrm{e}-005$ & 0.0106 & 0.0104 & 0.0309 & 0.0741 & 0.0077 \\
\hline $\begin{array}{c}\text { Contrast } \\
\text { Gain }\end{array}$ & 9.4662 & 7.3606 & 8.7331 & 8.7611 & 8.6608 & 5.3438 & 6.7696 \\
\hline VCM & 0.7264 & 0.4122 & 0.4252 & 0.4269 & -0.1904 & 0.2261 & -0.2097 \\
\hline$\Sigma$ & 0.5931 & 0.8452 & 0.6514 & 0.6503 & 0.6747 & 0.8820 & 0.7628 \\
\hline $\mathrm{HCC}$ & 0.4646 & 0.1820 & 0.3119 & 0.3123 & 0.3563 & 0.0523 & 0.3703 \\
\hline SSIM & 84.6667 & 66.6667 & 79.6667 & 79.8333 & 67.5000 & 55.1667 & 62 \\
\hline UQI & 0.8684 & 0.9618 & 0.9665 & 0.9666 & 0.7580 & 0.9835 & 0.7209 \\
\hline MSE & $\begin{array}{c}3.0776 \mathrm{e}+ \\
003\end{array}$ & 983.1221 & $\begin{array}{c}1.0129 \mathrm{e}+ \\
003\end{array}$ & $1.0137 \mathrm{e}+003$ & $3.8854 \mathrm{e}+003$ & 506.0890 & $\begin{array}{c}4.2552 \mathrm{e}+ \\
003\end{array}$ \\
\hline PSNR & 13.2487 & 18.2047 & 18.0753 & 18.0717 & 12.2365 & 21.0885 & 11.8416 \\
\hline NK & 0.8094 & 0.8522 & 0.9913 & 0.9907 & 0.7730 & 1.0489 & 0.6738 \\
\hline $\mathrm{AD}$ & 33.0683 & 25.3653 & 4.5679 & 4.6493 & 47.3099 & -4.4113 & 61.4883 \\
\hline $\mathrm{SC}$ & 1.3830 & 1.3556 & 0.9834 & 0.9845 & 1.4759 & 0.8969 & 2.0338 \\
\hline MD & 184 & 91 & 160 & 160 & 171 & 50 & 137 \\
\hline NAE & 0.2507 & 0.1639 & 0.1435 & 0.1435 & 0.3283 & 0.1128 & 0.3680 \\
\hline
\end{tabular}


All the codes and the quality assessment criterions have been developed and tested on the same computer. The system is Win 7 ultimate, MATLAB software 2010 $\mathrm{R}$; hardware is Intel Core i3 CPU and 4 GB RAM.

It has been observed that CLAHE is a simple and effective algorithm for enhancing dark and homogenous foggy images. On the other hand, CLAHE cannot solve depth discontinuous problem (Fig.4 fog2.jpg).

Meng et al. [48] is an improved version of He et al. [21], so its results are better but it causes color distortion problem (input image trains.bmp). Tarel and Hautiere[38] algorithm has achieved larger e value which means that it has the best performance in restoring image edges. Smaller the value of SSIM and UQI the better the defogging results. It also proves that removal of fog from an image will also change the image structure.

No defogging algorithm is best in all conditions, so it's difficult to say which one is the best algorithm. Fig.6 shows that the Tarel and Hautiere[38] algorithm greatly enhanced the road image with best results.

The DCP based defogging is the most popular single image defogging algorithm. From lake.jpg it has been observed that the guided image filtering can greatly enhance the efficiency.

\section{CONCLUSIONS}

This paper gives a brief introduction to image enhancement and restoration algorithms. The main problems in the related studies found are:

1. No algorithm can judge whether the image has fog or not. The existing algorithms can only restore a foggy image. The fog level classification is a worth studying.

2. No single image defogging algorithm is best suitable for all types of foggy weather. Present algorithms work well for homogenous fog or thin fog. It is the demand of the hour to establish an algorithm that can be suitable for all kinds of weather. Maybe we can use 2 or 3 techniques like image classification method, visibility detection and single image defogging methods for the same.

3. In some existing single image defogging algorithms, some parameters are required to be set manually which is unrealistic in real time applications. A fast, efficient and useful algorithm is the need of the hour.

4. It has been observed that objective image quality assessment methods are not consistent with subjective image quality assessment methods. Thus, some better image quality assessment method may be proposed. It may be some intelligent machine learning method like deep learning algorithm.

\section{REFERENCES}

[1] S. G. Narasimhan and S. K. Nayar, "Vision and the atmosphere,” Int. J. Comput. Vis., vol. 48, no. 3, pp. 233 254, 2002.

[2] T. El-gaaly, "Measuring Atmospheric Scattering from Digital Images of Urban Scenery using Temporal Polarization-Based Vision,” 2009.
[3] R. K. Thakur and C. Saravanan, "Classification of Color Hazy Images," no. August, 2016.

[4] X. Zhou, C. Wang, L. Wang, N. Wang, and Q. Fu, "Single Image Dehazing Using Dark Channel Prior and Minimal Atmospheric Veil," vol. 10, no. 1, pp. 341-363, 2016.

[5] "Contrast enhancement using real coded genetic algorithm based modified histogram equalization for gray scale images - Babu - 2015 - International Journal of Imaging Systems and Technology - Wiley Online Library."

[6] Xie, F. Guo, and Z. Cai, "Improved Single Image Dehazing Using Dark Channel Prior and Multi-scale Retinex," 2010 International Conference on Intelligent System Design and Engineering Application. pp. 848-851, 2010.

[7] S. G. Narasimhan and S. K. Nayar, "Contrast restoration of weather degraded images," IEEE Trans. Pattern Anal. Mach. Intell., vol. 25, no. 6, pp. 713-724, 2003.

[8] E. Namer and Y. Y. Schechner, "Advanced Visibility Improvement Based on Polarization Filtered Images," Proc. SPIE, Vol. 5888, pp. 36-45, 2005.

[9] Y. Y. Schechner, S. G. Narasimhan, and S. K. Nayar, "Polarization-Based Vision Through Haze," Appl. Opt., vol. 42, no. 3, p. 511, 2003.

[10] S. Shwartz, E. Namer, and Y. Y. Schechner, "Blind haze separation," Proc. IEEE Comput. Soc. Conf. Comput. Vis. Pattern Recognit., vol. 2, pp. 1984-1991, 2006.

[11] Y. Y. Schechner, S. G. Narasimhan, and S. K. Nayar, "Instant dehazing of images using polarization," Proc. 2001 IEEE Comput. Soc. Conf. Comput. Vis. Pattern Recognition. CVPR 2001, vol. 1, pp. 325-332, 2001.

[12] Miyazaki, D. Akiyama, M. Baba, R. Furukawa, S. Hiura, and N. Asada, "Polarization-based dehazing using two reference objects," Proc. IEEE Int. Conf. Comput. Vis., pp. 852-859, 2013.

[13] Y. Xu, S. Member, J. I. E. Wen, and L. Fei, "Review of Video and Image Defogging Algorithms and Related Studies on Image Restoration and Enhancement," pp. 165-188, 2016.

[14] S. G. Narasimhan, C. Wang, and S. K. Nayar, "All the Images of an Outdoor Scene," Comput. Vis. - ECCV 2002, vol. 2352, pp. 148-162, 2006.

[15] S. G. Narasimhan and S. K. Nayar, "Removing weather effects from monochrome images," Proc. 2001 IEEE Comput. Soc. Conf. Comput. Vis. Pattern Recognition. CVPR 2001, vol. 2, pp. 186-193, 2001.

[16] S. G. Narasimhan and S. K. Nayar, "Chromatic framework for vision in bad weather," Proc. IEEE Conf. Comput. Vis. Pattern Recognition. CVPR 2000 (Cat. No.PR00662), vol. 1, pp. 598-605, 2000.

[17] S. G. Narasimhan and S. K. Nayar, "Interactive ( De ) Weathering of an Image using Physical Models," IEEE Work. Color Photom. Methods Comput. Vis., pp. 1-8, 2003.

[18] S. K. Nayar and S. G. Narasimhan, "Vision in Bad Weather," Proc. Seventh IEEE Int. Conf. Comput. Vis., vol. 2, no. c, pp. 820-827 vol.2, 1999.

[19] R. Fattal, "Single image dehazing," ACM Trans. Graph., vol. 27, no. 3, p. 1, 2008.

[20] R. T. Tan, "Visibility in bad weather from a single image," 26th IEEE Conf. Comput. Vis. Pattern Recognition, CVPR, no. September, 2008.

[21] H. Kaiming, S. Jian, and T. Xiaoou, "Single Image Haze Removal Using Dark Channel Prior," IEEE Trans Pattern Anal Mach Intell, vol. 33, no. 12, pp. 2341-2353, 2011.

[22] W. De Dravo, "Dehazing with STRESS."

[23] Levin, D. Lischinski, and Y. Weiss, "A closed-form solution to natural image matting," IEEE Trans. Pattern 
Anal. Mach. Intell., vol. 30, no. 2, pp. 228-242, 2008.

[24] S. C. Huang, B. H. Chen, and W. J. Wang, "Visibility restoration of single hazy images captured in real-world weather conditions," IEEE Transactions on Circuits and Systems for Video Technology, vol. 24, no. 10. pp. 1814 1824,2014

[25] K. B. Gibson, D. T. Vo, and T. Q. Nguyen, "An Investigation of Dehazing Effects on Image and Video Coding," Tip, vol. 21, no. 2. pp. 662-673, 2012.

[26] K. Gibson, D. Vo, and T. Nguyen, "An investigation in dehazing compressed images and video," MTS/IEEE Seattle, Ocean. 2010, 2010.

[27] Park, D. K. Han, and H. Ko, "Single image haze removal with WLS-based edge-preserving smoothing filter," ICASSP, IEEE International Conference on Acoustics, Speech and Signal Processing - Proceedings. pp. 24692473, 2013.

[28] K. B. Gibson and T. Q. Nguyen, "Fast single image fog removal using the adaptive Wiener filter," 2013 IEEE Int. Conf. Image Process. ICIP 2013 - Proc., no. 2, pp. 714 $718,2013$.

[29] J. Yu, C. Xiao, and D. Li, "Physics-based fast single image fog removal," Int. Conf. Signal Process. Proceedings, ICSP, pp. 1048-1052, 2010.

[30] Xiao and J. Gan, "Fast image dehazing using guided joint bilateral filter," Vis. Comput., vol. 28, no. 6-8, pp. 713$721,2012$.

[31] K. He, J. Sun, and X. Tang, "Guided Image Filtering," Link.Springer.Com, vol. 6311, no. Chapter 1, pp. 1-14, 2010.

[32] K. He and J. Sun, "Fast Guided Filter," CoRR, vol. abs/1505.0, p. 2, 2015

[33] S. C. Pei and T. Y. Lee, "Nighttime haze removal using color transfer pre-processing and Dark Channel Prior," Proc. - Int. Conf. Image Process. ICIP, pp. 957-960, 2012.

[34] Z. Li, J. Zheng, Z. Zhu, W. Yao, and S. Wu, "Weighted guided image filtering," IEEE Trans. Image Process., vol. 24, no. 1, pp. 120-129, 2015.

[35] Z. Li and J. Zheng, "Edge-Preserving DecompositionBased Single Image Haze Removal," IEEE Trans. Image Process., vol. 24, no. 12, pp. 5432-5441, 2015.

[36] Z. Lin and X. Wang, "Dehazing for Image and Video Using Guided Filter,” pp. 123-127, 2012.

[37] Q. Zhu, J. Mai, and L. Shao, "A Fast Single Image Haze Removal Algorithm Using Color Attenuation Prior," IEEE Trans. Image Process., vol. 24, no. 11, pp. 3522 3533, 2015.

[38] J.-P. Tarel and N. Hautiere, "Fast visibility restoration from a single color or gray level image," Comput. Vis. 2009 IEEE 12th Int. Conf., no. Iccv, pp. 2201-2208, 2009.

[39] L. K. and K. Nishino, "Factorizing Scene Albedo and Depth from a Single Foggy Image," no. Iccv, pp. 1701$1708,2009$.

[40] K. Nishino, L. Kratz, and S. Lombardi, "Bayesian defogging," Int. J. Comput. Vis., vol. 98, no. 3, pp. 263 278, 2012.

[41] K. Tang, J. Yang, and J. Wang, "Investigating Hazerelevant Features in A Learning Framework for Image Dehazing."

[42] N. Hautière, J.-P. Tarel, D. Aubert, and É. Dumont, "Blind Contrast Enhancement Assessment By Gradient Ratioing At Visible Edges," Image Anal. Stereol., vol. 27, no. 2, pp. 87-95, 2008.
[43] X. Yu, C. Xiao, M. Deng, and L. Peng, "A classification algorithm to distinguish image as haze or non-haze," Proc. - 6th Int. Conf. Image Graph. ICIG 2011, no. 2, pp. 286$289,2011$.

[44] T. L. Economopoulos, P. A. Asvestas, and G. K. Matsopoulos, "Contrast enhancement of images using Partitioned Iterated Function Systems," Image Vis. Comput., vol. 28, no. 1, pp. 45-54, 2010.

[45] J. Jobson, "A comparison of visual statistics for the image enhancement of FORESITE aerial images with those of major image classes," Proc. SPIE, vol. 6246, pp. 624601624601-8, 2006.

[46] W. Chenyi and L. Guang, "The Latest Research Progress of Polyimides," no. 50673017, 2009.

[47] Z. Wang and A. Bovik, "A universal image quality index," IEEE Signal Process. Lett., vol. 9, no. 3, pp. 81-84, 2002.

[48] G. Meng, Y. Wang, J. Duan, S. Xiang, and C. Pan, "Efficient Image Dehazing with Boundary Constraint and Contextual Regularization," 2013 IEEE Int. Conf. Comput. Vis., pp. 617-624, 2013.

[49] B. Cai, X. Xu, K. Jia, C. Qing, and D. Tao, "DehazeNet: An End-to-End System for Single Image Haze Removal," pp. 1-11, 2016.

\section{Authors' Profiles}

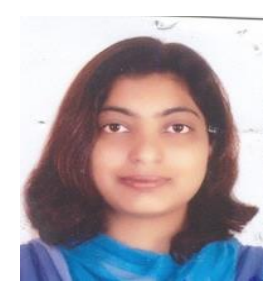

Bindu Bansal received B.Tech. Degree in Computer Science and Engineering from Punjab Technical University, Jalandhar in 2005 and completed her M.Tech. Degree in Computer Science and Engineering from Punjab Technical University, Jalandhar in 2012. She has 8 years of teaching experience and 3 years of administration experience. She is also a life-member of ISTE. She is pursuing Ph.D. from IKG-PTU Jalandhar in the area of Image / Video Processing. She guided various graduate/post graduate projects. She has interest in the databases, image processing.

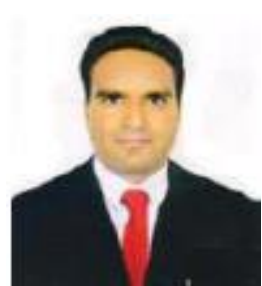

Prof. (Dr.) Jagroop Singh Sidhu completed his Bachelor's degree from Sant Longowal Institute of Engineering and Technology, Longowal (Punjab), India and Masters degree in Electronics and Communication Engineering with Hons. (2001) from Thapar Institute of Engineering and Technology, Patiala. He has obtained his PhD degree in the field of Image Processing from National Institute of Technology, Jalandhar, Punjab, India. Presently, he is serving as an Associate Professor in the Department of Electronics and Communication Engineering, at DAVIET, Jalandhar, Punjab, India. He has more than 38 research publications in internationally reputed journals and conference proceedings. His research interests include image compression, telemedicine and Signal and Image Processing.

Dr. Kiran Jyoti is Associate Professor in GNE Ludhiana, Punjab 
How to cite this paper: Bindu Bansal, Jagroop Singh Sidhu, Kiran Jyoti," A Review of Image Restoration based Image Defogging Algorithms", International Journal of Image, Graphics and Signal Processing(IJIGSP), Vol.9, No.11, pp. 6274, 2017.DOI: 10.5815/ijigsp.2017.11.07 